team has modelled ${ }^{1}$ how colorectal cancers respond to targeted therapies that are given in combinations, potentially revealing ways to prevent the tumour cells from becoming resistant. "We have very exciting data now on the possibility to track and treat evolution," he says.

\section{TREE OF LIFE}

Cancer cells harbour a staggering array of mutations. In 2012, when Swanton and his colleagues sequenced multiple biopsies from two people with kidney cancer, they found that even within a single person, no two samples were the same ${ }^{2}$. The team examined not only the primary tumour, but also the satellite tumours - called metastases - that had spread throughout the patients' bodies. In each person, the team found more than 100 mutations in the various tumour samples analysed; only about one-third of them occurred in all samples.

The relationships between the various cancerous cells from a single person can be plotted out in much the same way as evolutionary biologists plot relationships between species: by drawing phylogenetic trees, branching diagrams that trace 'descendants' back to a common ancestor. Mutations that occur in the first malignant cells, those in the trunk of this evolutionary tree, will end up in all the tumour cells; mutations that arise later will be found only in the tree's branches. To eliminate the tumour, Swanton says, one must attack the mutations in the trunk.

Therapies that target some of these trunk mutations already exist, and they often produce dramatic responses at first. But then resistance develops, as Bardelli found. "We're so fixated on 'the smaller the tumour gets, the better', but what one doesn't think about is what one has left behind," Swanton says. "You're often leaving resistant clones that you can't treat." But he thinks that by targeting multiple trunk mutations at the same time, researchers might have a shot at wiping out the cancer. Chances are slim that a single cancer cell would be able to evade a two- or threepronged attack.

One way to do this is to use combinations of targeted therapies. "In theory, they can work," says Bert Vogelstein, a cancer geneticist at the Sidney Kimmel Comprehensive Cancer Center at Johns Hopkins University in Baltimore, Maryland. In fact, when he and evolutionary biologist Martin Nowak at Harvard University in Cambridge, Massachusetts, modelled the strategy, they found that two targeted medicines for which no common resistance mechanism exists would be enough to control metastatic cancer ${ }^{3}$. For people with a large number of metastases, the model suggested that three therapies would be needed.

Researchers are already beginning to test combinations of targeted therapies in the clinic. However, Swanton points out that there are no targeted drugs for the vast majority of mutations. And combining existing drugs in a way that won't harm the patient has proved tricky. So Swanton is focusing on immunotherapies - strategies that help the immune system to recognize and destroy cancer cells (see page 162).

The immune system identifies threats, in part, by surveying the surfaces of cells, looking for molecules called antigens that can signal

\section{"CANCER ISCONTINUOUSLY ADAPTING, THEREFOREWE HAVETODO SO ASWELL."}

trouble within. The genetic defects in the DNA of a cancer cell can sometimes encode antigens that will trigger an immune response. But Swanton and his colleagues wondered whether it matters if the immune system responds to an antigen that arises from the cancer's evolutionary trunk or to one that springs from its branches.

In a paper published in $\mathrm{March}^{4}$, he and his colleagues examined samples from the Cancer Genome Atlas, a collection of genetic and clinical data from thousands of people with cancer. They found that people with lung cancer who had lots of trunk antigens - and a high proportion of trunk antigens to branch antigens - survived longer than those who had either few trunk antigens or a higher proportion of branch antigens. What's more, people with many trunk antigens seemed to respond better to immune therapies. That makes sense, Swanton says, because if the immune system targets trunk antigens, it's hitting most of the cancer cells, rather than "nipping off little branches".

The research is still in its infancy, but Swanton is leading a clinical study that could help to confirm his findings. The study, called TRACERx - Tracking Cancer Evolution through Treatment $(\mathrm{Rx})$ - will follow 850 people from lung-cancer diagnosis through treatment and, in some cases, until death. It will document genetic changes in their tumours over time to examine how lung cancer evolves, and how treatment influences that process.

Once he has the data, Swanton hopes to raise enough money to test treatment strategies based on evolution. One approach would be to identify immune cells in a tumour, grow them in a lab, and then infuse them back into the patient - a technique called adoptive cell transfer. Similar strategies already in use select immune cells that recognize any cancer antigen, but Swanton's group would select those that are primed to recognize the trunk antigens that occur on all cancer cells.

This strategy would not be cheap, but neither is doling out a string of targeted therapies only to watch them all eventually fail. "Each course of therapy costs between US $\$ 10,000$ and $\$ 100,000$," Swanton says. If researchers could develop a therapy that would cure metastatic cancer, "the whole cost-benefit analysis and the health economic models change dramatically".

\section{CELLULAR COMPETITORS}

Applying evolutionary principles might help the immune system to vanquish tumours. Robert Gatenby, a molecular oncologist at Moffitt Cancer Center in Tampa, Florida, has a more modest goal: he hopes to help people to live with their disease. Gatenby began thinking about cancer as an evolutionary problem in the early 1990s, when he was working at Fox Chase Cancer Center in Philadelphia, Pennsylvania. He saw so many people relapse that cancer began to seem less like a biological problem and more like witchcraft. "It's like an evil entity that just keeps recurring and overcoming your best efforts." But when he began thinking about cancer from an evolutionary perspective, the problem became tractable again, he says.

Gatenby began trying to mathematically model the disease to work out how best to tackle it. His models suggest that many oncologists are taking the wrong approach. Typically, physicians will give the maximum dose of chemotherapy that a person can tolerate, to kill as many cancerous cells as possible. The hope is that they can wipe out the cancer before resistance evolves.

But studies from recent years suggest that tumours harbour drug-resistant cells long before they encounter therapy ${ }^{5-7}$. The population of resistant cells stays small because resistance comes with a fitness cost. When a patient receives a hefty dose of chemotherapy, however, the resistant cells become much fitter than the susceptible cells. Gatenby likens drug resistance to an umbrella: "If it's raining, the umbrella is very useful. But if it's not raining, it's a burden." Gatenby thinks that he can capitalize on the natural competition between susceptible and resistant cells by managing drug dosage or timing more carefully.

Recently, he tested the idea in mice with two kinds of breast cancer ${ }^{8}$. When he and his colleagues gave the mice the standard, maximum tolerated dose of the chemotherapy drug paclitaxel, the tumours roared back as soon as the treatment was stopped. The team also tried skipping doses whenever the tumour began to shrink, but that worked no better. A third group of mice received the standard high dose of chemotherapy at first, but once the animals' tumours started to shrink, the researchers dialled back the dose. This strategy resulted in the best survival for the mice and allowed three out of the five mice tested to be weaned off the 
drug completely. The treatment is meant to adapt to how the tumour responds and maintain a balance between drug-resistant and susceptible cells (see 'Evolving strategies'). "I think it's one of the most exciting advances in cancer biology because it's a relatively easy thing to try," says Carlo Maley, a biologist at Arizona State University in Tempe who has collaborated with Gatenby.

In May 2015, the Moffitt Cancer Center launched a pilot study to test whether this kind of adaptivetherapy approach might help people with prostate cancer. Physicians will monitor the patient's levels of prostate specific antigen (PSA), a marker of disease progression. They will then administer standard treatment or stop it, depending on what they see. Researchers have studied intermittent therapy in the past, but the protocols generally involve rigidly controlled cycles. "With adaptive therapy, the on-off cycle is determined by the tumour response," Gatenby says. He also plans to use the wealth of molecular and clinical data from the trial to develop computer models that might guide adaptive therapy in the future.

\section{IN A BIND}

Physicians have noticed other evolutionary paradigms at work. In January, Jeffrey Engelman, a thoracic oncologist at Massachusetts General Hospital in Boston, and his colleagues detailed the case of a 52-year-old woman with metastatic lung cancer in The New England Journal of Medicine ${ }^{9}$. The woman's tumour had a genetic rearrangement that produced a misshapen version of the ALK protein, so her doctors first administered the drug crizotinib, which inhibits the action of ALK. She responded well for 18 months, but then relapsed. A second-generation therapy also failed, so physicians moved onto a thirdgeneration therapy that is still in clinical trials. That worked for a while, but after less than a year, the woman's liver began to fail, and she had to be hospitalized. Then her doctors found that the third therapy had prompted a new mutation that made her cancer once again responsive to crizotinib. When they administered the drug, her liver recovered, and she improved so much that she was able to leave the hospital.

For Engelman and his colleagues, the woman's resensitization to crizotinib was a happy accident. But researchers may be able to direct cancer down such routes intentionally. Gatenby calls this strategy an evolutionary double bind, and he explains it like

\section{EVOLVING STRATEGIES}

Stemming tumour growth

As cancer cells divide,

new mutations emerge,

stablishing new cell

opulations that can

e mapped on an

volutionary tree.

A therapy that

targets mutations

has a better chance

of eliminating

ancer.
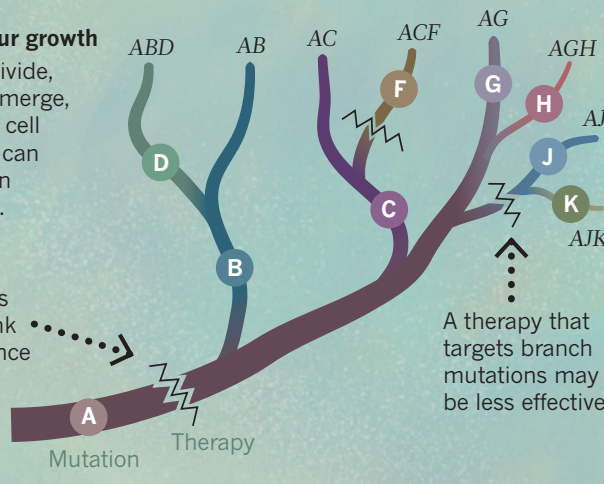

Adapting for balance

Cancer-cell populations compete, so completely killing cells that are sensitive a particular drug lets resistant cells grow unfettered. Adjusting dosage

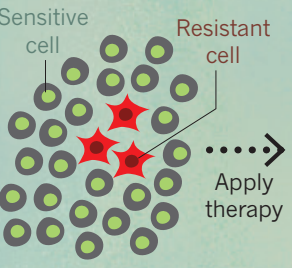

nly some cells are resistant.

\section{Some sensitive} cells remain.

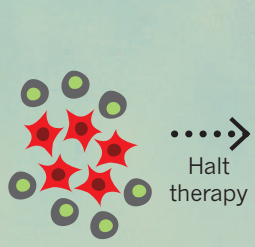

The tumour remains treatable.
The double bind

Developing resistance to one treatment can leave tumours vulnerable others. Evolutionary modelling can suggest the best way to apply Apply Sensitive to therapy 1

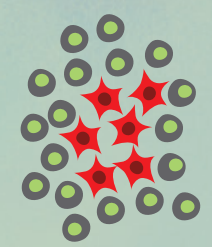

(n)

\section{if the trials fail, the results of the tests} will help researchers to refine their theories, and will address some of the big unknowns. How do the genetically diverse cells in a tumour interact, for example, and what is the role of the cellular environment that they inhabit? Kornelia Polyak, an oncologist at Harvard Medical School in Boston, says that cancer researchers tend to focus on the mutations inside cells, and fail to consider how those mutated cells might influence the cells around them. "That's a largely

this: imagine trying to control a population of rats by introducing predators, such as hawks, that can pick them off from the sky. That type of predation selects for rodents that hide under brush. So one might bring in snakes that also hide under brush. The snakes would select for rats that prefer open spaces, making them vulnerable to hawks, Gatenby says. The same idea could apply in cancer. Use one treatment that makes the cancer more vulnerable to a second one, and then alternate between the two. It's "not whack-a-mole", Gatenby says, "but rather a carefully thought-out method that exploits the evolutionary dynamics".

That strategy is exactly what Ben Solomon, a cancer researcher at the Peter MacCallum Cancer Centre in Melbourne, Australia, plans to test in an upcoming trial. Many people with lung cancer harbour mutations in a gene called EGFR. Several drugs have been approved to target EGFR mutations, but tumours invariably unexplored area," she says.

The dynamics inside a tumour are exceedingly complicated, but Engelman is not discouraged. Clinical analyses will help researchers to understand this complexity. "These insights are going to bring us closer to having bigger and bigger impacts," he says. "The depressing thing is to not know what the hell is going on."

Cassandra Willyard is a science writer based in Madison, Wisconsin.

1. Misale, S. et al. Nature Commun. 6, 8305 (2015).

2. Gerlinger, M. et al. N. Engl. J. Med. 366, 883-892 (2012).

3. Bozic, l. et al. eLife 2, e00747 (2013).

4. McGranahan, N. et al. Science 351, 1463-1469 (2016).

5. Turke, A. B. et al. Cancer Cell 17, 77-88 (2010).

6. Bhang, H. C. et al. Nature Med. 21, 440-448 (2015).

7. Su, K. Y. et al. J. Clin. Oncol. 30, 433-440 (2012).

8. Enriquez-Navas, P. M. et al. Sci. Transl. Med. 8, 327ra24 (2016).

9. Shaw, A. T. et al. N. Engl. J. Med. 374, 54-61 (2016). 